\title{
Probleme der Identitätskonstitution im interkulturellen Spannungsfeld am Beispiel von Güney Dals Der enthaarte Affe
}

WEERTJE Willms

\begin{abstract}
This article analyzes the problems of identity in the novel Der enthaarte Affe by the Turkish-German author Güney Dal by taking into account the intercultural dimension and psychological insight. The protagonist's identity problems rise from a loss of balance between the way he sees himself and the way he is seen by others; he suffers from several psychic and psychosomatic diseases, which are connected with his status as a foreigner. The doppelgänger constellation on the level of narrative fiction and diegetic confusion can be interpreted as a sign of the protagonist's split personality. In fact, on the level of fictional action, it is impossible to find out which doppelgänger figure is real and which one is imagined. This impossibility can be regarded as a fictional correlate of the protagonist's split personality.
\end{abstract}

\section{EinLeitung}

Als mit dem Anwerbevertrag für die sogenannten Gastarbeiter Anfang der $1960 e r$ Jahre türkische Arbeiter nach Deutschland kommen, entstehen die ersten Texte von Türken in Deutschland - in Form von Briefen, Gedichten oder Liedern, welche die Erfahrungen der Migration und der deutschen Arbeitswelt verarbeiten (Yeşilada 2008). Die vor allem etwas später emigrierenden Intellektuellen entwickeln ein Interesse für die türkische Arbeitsmigration und die Integration der Emigranten und setzen sich dafür ein, dass kulturelle Zusammenschlüsse und Publikationsorgane entstehen, wodurch eine Kulturvermittlung zwischen den türkischen Autoren und der deutschen Öffentlichkeit beginnt (vgl. Weigel 1992). Die Texte der türkischen Auswanderer kreisen in diesen ersten Jahren stets um Themen, die in unmittelbarem Zusammenhang mit der 
Emigration auf Zeit und der Arbeit in Deutschland stehen, z.B.: der Wechsel vom Dorf in die (Groß-)Stadt, die entfremdete Arbeit in der Fabrik, die Rechtlosigkeit und die Ausbeutung der Arbeiter, die Sehnsucht nach der Heimat und der dort verbliebenen Familie, die Einsamkeit in der Fremde und die Isolation durch mangelnde Sprachkenntnisse, die Ausländerfeindlichkeit bei vielen Deutschen, das Erstarken traditioneller Werte wie Religion und Familienzusammenhalt in der Fremde, der >Gastarbeitertraum < von Geldsparen und Rückkehr. Aufgrund der thematischen Anbindung dieser neu entstehenden Literatur an den Erfahrungsraum der sogenannten Gastarbeit und deshalb, weil die Autoren selbst > Gastarbeiter < sind (die meisten indes nur vorübergehend, da sie - bis auf wenige Ausnahmen ${ }^{1}$ - Intellektuelle sind, die bald wieder in akademische bzw. künstlerische Berufe wechseln), erhalten die Texte die Bezeichnung »Gastarbeiterliteratur «; eine Bezeichnung, von der sich die meisten Forscherinnen und Forscher heute allerdings strikt distanzieren. ${ }^{2}$ Der Stil dieser Texte ist schlicht, er gemahnt an Erfahrungsberichte oder an Reportagen aus der Arbeitswelt, welche in den 1970er Jahren in der Bundesrepublik Konjunktur haben.

Ende der 1980er Jahre findet eine Veränderung in der Literaturproduktion türkischer Autoren in Deutschland statt, welche vor allem durch das Erscheinen von zwei Romanen im Jahr 1988 markiert ist: Aras Örens Eine verspätete Abrechnung oder der Aufstieg der Gündoğdus und Güney Dals Der enthaarte Affe, 3 dem ich

1 | Der bekannteste wirkliche Arbeiterschriftsteller ist Fethi Savaşçı.

2 | In der Forschung wird heute der Begriff "Gastarbeiterliteratur" fast durchweg abgelehnt, da er als "patriarchalisch", "herablassend" und "unverschämt" empfunden wird und die Literaturproduktion der ersten Autorengeneration »in sozialpädagogischer Fürsorge lediglich als Dokument der Betroffenheit betrachtet", wie Hofmann $(2006,201)$ es ausdrückt. Kreuzer spricht sich 1984 noch für den Begriff aus, weil die Rezipienten die unter diesen Terminus subsumierte Literatur leicht einordnen könnten, da er eine bestimmte Zeit und einen bestimmten geografischen Raum bezeichne (nämlich die Bundesrepublik der 1970er Jahre) und somit spezifischer sei als beispielsweise ein Begriff wie "Migrantenliteratur". Außerdem lasse sich dieser Terminus, der die literarische Produktion verschiedener ethnischer Gruppen umfasst, an den Begriff der "Arbeiter-Literatur" anschließen, der in den 1970er Jahren in der Bundesrepublik wichtig war. Nach zahlreichen Vorschlägen wie z.B. "Ausländerliteratur" (Ackermann/Weinrich 1986), "kleine Literatur" (Weigel 1992), "deutschsprachige Minderheitenliteratur" (Karl Esselborn), "Migrantenliteratur", "Migrationsliteratur" wird heute von vielen der Terminus "interkulturelle Literatur" (z.B. Chiellino 2007) vorgezogen. Meines Erachtens schränkt dieser Terminus die darunter subsumierten Texte indes zu sehr auf eine angenommene interkulturell ausgerichtete Thematik ein, was zwar vielen, aber längst nicht allen Texten entspricht, die von Autorinnen und Autoren nicht-deutscher Herkunft geschrieben werden (vgl. z.B. die Texte von Akif Pirinçci und Selim Özdoğan). Ich verwende daher die Bezeichnung türkisch-deutsche Literatur bzw. Autoren, sofern die Hervorhebung der nationalen Herkunft des Autors oder der Autorin für den Text von Bedeutung ist, da diese pragmatisch gemeinte Bezeichnung keine thematische Eingrenzung der Texte schafft.

3 | Elf Jahre später ist der Text unter dem Titel "Janitscharenmusik" mit einigen wenigen Veränderungen erneut erschienen. 
meine Aufmerksamkeit im Folgenden widmen möchte. Die Texte dieser - literaturgeschichtlich - zweiten Generation von Autoren wenden sich sowohl neuen Themen als auch neuen Ausdrucksformen zu. Es werden Fragen der Integration in die deutsche Gesellschaft und der nun im Spannungsfeld von zwei Kulturen zu verortenden Identität wichtig; zumal dann, wenn die Familie nach Deutschland nachgezogen ist und auch die Kinder der Gastarbeiter in der deutschen Gesellschaft einen Platz finden müssen. Sind die Texte der ersten Generation auffällig schlicht gestaltet (s.o.), so stellen die der zweiten Generation interessanterweise die Literarizität in besonderer Weise in den Vordergrund. Die Reportage über Arbeitswelt-Erfahrungen scheint von der interkulturellen Auseinandersetzung abgelöst $\mathrm{zu}$ werden, welche ganz bewusst die literarische Stilisierung und Verfremdung als Ausdruckmittel wählt. Dabei verändert sich auch der Blickwinkel der Erzähler: Handelt es sich zunächst um den Blickwinkel der Türken auf das fremde Deutschland bzw. der Türken, die eine Identität in Auseinandersetzung mit zwei Kulturen entwickeln müssen, finden die jüngeren Autorengenerationen zu einer übernationalen, kosmopolitischen Betrachtungsweise, die dann in den jüngsten Texten sogar von einem eher >deutschen < Blickwinkel abgelöst wird.

Der hier im Zentrum stehende Autor Güney Dal repräsentiert in seinem Lebenslauf zahlreiche >typische< Merkmale türkisch-deutscher Autoren seiner Generation: Dal wird 1944 in der Türkei geboren und emigriert 1972 - also als erwachsener Mann von 28 Jahren - nach Deutschland, nachdem er in seinem Heimatland ein Studium absolviert und in verschiedenen kulturell orientierten Berufen gearbeitet hat (z.B. als Buchhändler und Journalist). In Deutschland arbeitet Dal zunächst als Fabrikarbeiter ( $>$ Gastarbeiter $<$ ), später als Journalist und Autor für den Sender Freies Berlin. Ende der 1970er Jahre lässt er sich als freier Autor nieder und ist inzwischen mit zahlreichen Preisen ausgezeichnet worden. Der enthaarte Affe ist Dals dritter Roman, der - wie alle Texte dieses Autors - auf Türkisch geschrieben ist und in engem Kontakt mit dem Übersetzer Carl Koß ins Deutsche übertragen wurde. Anders als die Texte von Emine Sevgi Özdamar und Feridun Zaimoğlu ist Dals Roman nicht sehr bekannt geworden. Sowohl in Bezug auf die Erzählverfahren als auch hinsichtlich der in ihm verhandelten Themen ist er indes ein bedeutender und wichtiger Text, dem eine Schlüsselposition in der Entwicklung der türkisch-deutschen Literatur in Deutschland zukommt.

\section{IDENTITÄT UND INTERKULTURALITÄT in Dals DeR enthatarte Affe}

Der Text ${ }^{4}$ besteht aus zwei Teilen, der erste mit der Überschrift Zeitungstage (EA, 1-205) betitelt, der zweite mit der Überschrift Aus den Notizen eines Spions (EA, 217-350). Der erste Teil wird von dem Ich-Erzähler Ömer Kul, einem in Berlin lebenden türkischen Gastarbeiter, erzählt, welcher sich krankschreiben lässt, um

4 I Im Folgenden abgekürzt durch "EA" im Fließtext. Alle Seitenzahlen beziehen sich auf die in der Bibliografie angegebene Ausgabe von 1988. 
ein Buch zu verfassen. Er ist seit Jahren davon überzeugt, dass fast alle journalistischen Meldungen Betrug seien, und er hat die seiner Ansicht nach eigentlichen >Wahrheiten < über die Welt in Form von Notizen gesammelt, welche er nun zusammenstellen möchte. Hierfür wählt er die Form einer Wandzeitung, die er nachts schreibt und tagsüber von seiner Familie lesen lässt. In den Zeitungstagen berichtet Ömer tagebuchartig von dem Verfassen und Erscheinen der Wandzeitung. Gleichzeitig manifestiert sich bei ihm eine psychische Krankheit, die im Verlauf der Handlung immer weiter voranschreitet, bis er von seiner Familie in eine Klinik eingeliefert wird, womit der erste Teil des Romans endet. Als Ömers Wahn schon relativ ausgeprägt ist, findet er seine eigenen Notizen in der Schreibtischschublade, erkennt sie jedoch nicht wieder und interpretiert sie stattdessen als die Notizen eines Spions, dem er unterstellt, in sein Haus eingedrungen zu sein und ihn zu bedrohen. Diese Notizen eines Spions bilden den zweiten Teil des Romans, in dessen Mittelpunkt Ibrahim Yaprak steht - ebenfalls ein Türke in Berlin mit zahlreichen Parallelen zu Ömer. Ibrahims Geschichte wird in Du-Form berichtet, und er wird als ein Schriftsteller mit einer Schreibblockade vorgestellt, der nach vielen Jahren endlich seinen Roman schreibt, und zwar über einen türkischen Gastarbeiter in Berlin namens Ömer. Der erste Teil des Romans wird nun als das Romanmanuskript Ibrahims - mit Ömer als dessen Hauptfigur - dargestellt, so dass sich das Verhältnis zwischen Ömer und Ibrahim umdreht: Ist zunächst Ibrahim (als namenloser Spion) eine imaginierte Figur Ömers, wird nun Ömer zu einer imaginierten Figur Ibrahims.

Im Folgenden konzentriere ich mich auf die Identitätsproblematik, welche ich unter interkulturellem Gesichtspunkt analysieren möchte, das heißt, ich möchte zeigen, inwieweit die Identitätsproblematik mit der Migrationserfahrung und der Auseinandersetzung mit der fremdkulturellen Umgebung im Zusammenhang steht, wobei ich auf Theorien und Erkenntnisse aus der Psychologie zurückgreife. Außerdem untersuche ich, welche Rolle in diesem Zusammenhang die Vermittlungsebene spielt.

\subsection{Manifestationen des Wahnsinns beim Ich-Erzähler}

Der autodiegetische Erzähler und Protagonist des ersten Teils des Romans, Ömer Kul, präsentiert uns alle Ereignisse aus seiner Perspektive. Es gibt hier keinen ordnenden Eingriff eines anderen, übergeordneten Erzählers, stattdessen präsentiert sich uns weitgehend ein innerer Monolog, bei dem Erleben und Erzählen der intern fokalisierten Erzählerstimme fast deckungsgleich sind. Hierdurch entsteht eine große Unmittelbarkeit des Wiedergegebenen, wir tauchen ein in die Welt von Ömer, in seine Problematik, in seine wahnhafte Störung. Dennoch liefert Ömer so viele Informationen über das Handeln, das Verhalten und die Aussagen anderer Figuren, dass wir als Rezipienten einen Abgleich mit der Text-Realität erhalten und die geschilderten Ereignisse unabhängig von Ömers Interpretation verstehen können.

Solange der Erzähler und Protagonist Ömer zu Beginn des Textes noch in den geordneten Verhältnissen von Arbeit, Kaffeehaus und Familie lebt, ist sein 
Wahn gebändigt. In dem Moment aber, als er sich krankschreiben lässt, den ganzen Tag zuhause ist und sich mit seiner paranoiden Wahnvorstellung nämlich die angeblichen Lügen böser Mächte zu sammeln und aufzuschreiben - beschäftigt, wird seine Identitätsproblematik manifest, welche mit folgenden Krankheitsbildern aus der Psychiatrie umrissen werden kann: Fehleinschätzungen der Realität und des eigenen Verhaltens, Verfolgungswahn, Größenwahn, Zwangsneurose, fehlende Empathiefähigkeit und Verflachen der Affekte, psychosomatische Beschwerden, Halluzinationen und Persönlichkeitsspaltung. Diese Krankheitszustände - welche übrigens in der Psychologie unter den Begriff »Migrationspsychose« subsumiert werden (vgl. Black 1993, 160; Kora 1998) - prägen sich immer weiter aus, bis Ömer körperlich zusammenbricht und keinerlei Bezug mehr zur Realität hat. Sein grundlegender Identitätskonflikt - sowohl unter psychologischem Gesichtspunkt betrachtet als auch unter literarischem, also bezogen auf den Roman als Erzählkonstrukt - ist derjenige der Persönlichkeitsspaltung und des Wahns.

Bereits die erste Ausgabe der Wandzeitung macht offensichtlich, dass Ömer eigentlich gar nichts zu sagen hat. Er steigert sich immer weiter in die Welt seiner Zeitung hinein, die zu einer sich verselbständigenden Realität für ihn wird. Er fungiert als der Herausgeber und Redakteur der Zeitung und trennt dabei deutlich zwischen sich und dem Ömer der Zeitung, nimmt diesen also als real existent an, wie in einer Aussage gegenüber seiner Tochter deutlich wird: »Es ist hier nicht die Rede von deinem Vater Ömer Kul, es handelt sich vielmehr um einen der wenigen Korrespondenten der Zeitung, die du dort siehst, mit Namen Ömer Kul. Das heißt, es ist ein anderer Ömer Kul.« (EA, 79) Diese Persönlichkeitsspaltung wird später noch manifester, als Ömer sich in viele verschiedene Korrespondenten namens Ömer Kul aufspaltet und am Ende sogar der alleinige Gegenstand der Nachrichten selbst wird. Die von ihm repräsentierten Korrespondenten besitzen stets einen außergewöhnlichen Status (amerikanischer Präsident, KZ-Arzt, Professor für Genforschung u.a.), die Nachrichten über sich selbst mutieren gar zu einer >Selbstbeweihräucherung< (z.B. EA, 197). Ömer hält seine Zeitung und seine >Wahrheiten ` für eine Aufgabe von nationaler Bedeutung, für die er von allen höchste Aufmerksamkeit und Bewunderung verlangt, am Ende wähnt er sich gar in göttlicher Nähe:

Einmal ging mir [...] der Gedanke durch den Kopf, daß mir eine göttliche Offenbarung zuteil werden könnte. [...] [Auf eine Nachfrage der Tochter:] "Ich habe für einen Moment geglaubt, daß ich Gottes Prophet werden würde, mein Schatz“ [...]. (EA, 110)

Dieser extreme Größenwahn ist jedoch nur die Kehrseite des Minderwertigkeitskomplexes, den Ömer als Arbeiter und als Ausländer in Deutschland hat. Eher versteckt gibt er immer wieder zu verstehen, dass seine Zeitung die »Stimme der schweigenden Millionen« $(E A, 192)$ von Arbeitern sei. Die dunklen Mächte dagegen, welche die Wahrheiten verfälschten, rekrutierten sich aus den kapitalistischen Großverdienern und den Mächtigen in der Gesellschaft, welche die Arbeiter unterdrückten, ausnutzten und betrögen. Das Gefühl, ein rechtlo- 
ser ausländischer Arbeiter zu sein, das Gefühl der völligen Nichtigkeit und der fehlenden Verankerung im Lebensumfeld äußert sich in dem Größenwahn, es >denen da oben mal zu zeigen .

Die Aufspaltung in die verschiedenen Zeitungskorrespondenten deutet bereits an, dass Ömer in Bezug auf seine Identität verunsichert ist, was vor allem in seiner Doppelgänger-Fantasie manifest wird, welche schon zu Beginn des Textes eingeführt wird und die Struktur des gesamten Romans prägt. Ömer beschreibt den Beginn seiner Persönlichkeitsspaltung folgendermaßen:

Jedesmal hatte ich Angst vor diesem Gefühl und versuchte es zu verdrängen, als ob ich es nicht erlebt hätte. [...] Sie trat zuerst auf, als ich ungefähr zehn Jahre in Berlin war, diese Traum-Wirklichkeit: Von Zeit zu Zeit lebt neben mir, Schulter an Schulter, ein anderer Ömer Kul, er atmet, er macht immer genau das, was ich gerade mache, oder versucht mich zu ärgern, indem er das genaue Gegenteil macht. Ein solches Hirngespinst eben. (EA, 59f.)

Aus den nun folgenden Berichten, in denen der >andere Ömer Kul< ein immer stärkeres Gewicht einnimmt, wird deutlich, dass es sich hierbei um einen halluzinierten Doppelgänger handelt, also um eine (weitere) wahnhafte Persönlichkeitsspaltung Ömers. Entgegen der einsichtig wirkenden eben zitierten Schilderung nimmt Ömer seinen Doppelgänger jedoch im weiteren Verlauf als einen real existierenden fremden Mann wahr, dessen Existenz immer größeres Gewicht einnimmt. Ömer wundert sich über die Parallelen zwischen ihm und dem »lügenhafte[n], vaterlandslose[n] Bandit[en]« $(E A, 142)$, von dem er in Folge annimmt, dass er ein Agent oder Spion sei, der von der Regierung oder der Armee auf ihn und seine Familie angesetzt worden sei. Er unterstellt diesen Mächten, ihn ausschalten zu wollen, um weiter die Wahrheiten in der Welt verdrehen und Profit machen zu können.

Bevor ich auf die Doppelgängerproblematik und ihre Manifestation auf der Ebene der Romanstruktur weiter eingehe, soll die Identitätsproblematik Ömers in Bezug auf sein Verhältnis zu Deutschland näher untersucht werden.

\section{2 Ömers Verhältnis zu Deutschland Die Problematik von Eigen- und Fremdwahrnehmung}

Obwohl Ömer, wie er an einer Stelle seiner Aufzeichnungen erwähnt, seit 20 Jahren in Deutschland lebt, hat er keinen persönlichen Kontakt zu Deutschen. Dennoch steht er in einem extremen Spannungsverhältnis zu der Wahrnehmung der Deutschen und den ihnen zugeschriebenen Werten und Charaktermerkmalen. Dabei wird deutlich, dass sein Selbstbild insofern gestört ist, als er zu keinem ausbalancierten Verhältnis von Eigenwahrnehmung und antizipierter Fremdwahrnehmung gelangt. ${ }^{5}$ Stattdessen entwickelt Ömer eine

5 | Nach der Terminologie von George Herbert Mead (1973) sind die Instanzen von "ICh" ("|") und "ICH" ("Me"), welche das Subjekt zu einem Selbstkonzept integrieren 
ausgeprägte Paranoia. Unter anderem ist er von der zwanghaften Vorstellung besessen, von den Deutschen stets und ausschließlich als Ausländer wahrgenommen zu werden. Die antizipierte oder unterstellte Fremdwahrnehmung hat in Ömers Selbstkonstitution das Übergewicht bekommen und ist so sehr zu einer ihn verfolgenden fixen Idee geworden, dass sie in Form eines Ausbrechens von Unterdrücktem sich immer wieder Bahn bricht. Wenn Ömer mit Deutschen zusammen ist, thematisiert er zwanghaft von sich aus seinen Ausländerstatus und ist außerdem davon überzeugt, dass er deswegen verachtet wird (EA, 153).

Ömer scheint sich mit der unterstellten Fremdwahrnehmung so sehr zu identifizieren, dass er sich sogar die vermeintlich >deutsche Wahrnehmung zu eigen macht. Dies hat einerseits zur Folge, dass er, statt sich mit den eigenen Landsleuten zu solidarisieren, sich von diesen distanziert und sie kritisiert (z.B. EA, 18), sich also auf die Seite der ausländerfeindlichen Deutschen stellt. Zum anderen bewirkt diese Internalisierung der Fremdwahrnehmung und die Identifizierung mit ihr, dass er vor sich selbst keine Kritik an den Deutschen zulässt, was ihn immer wieder in einen Zwiespalt zwischen Wahrnehmung und Bewertung des Erlebten bringt. Ömer scheint schlechte Eigenschaften der Deutschen oder Probleme, die in Deutschland existieren, zwar durchaus wahrzunehmen, er erwähnt sie in seinen an einen fiktiven Leser gerichteten Aufzeichnungen auch, doch schafft er es nicht, die Dinge beim Namen zu nennen und Konsequenzen daraus zu ziehen. Entsprechend begegnet Ömer - der seiner eigenen Familie gegenüber rücksichtslos und tyrannisch ist - den Deutschen mit Unterwerfungsgesten, Überfreundlichkeit und Hyperkorrektheit. Man kann nicht sagen, dass er in Deutschland integriert wäre - er bewegt sich in einem rein türkischen Umfeld -, auch von Assimilation zu sprechen, wäre der falsche Begriff. Es handelt sich eher um eine Art Überidentifizierung mit den Deutschen, welche ihn jedoch ebenso von den türkischen Landsleuten in Berlin distanziert wie auch von den zurückgebliebenen Verwandten und Freunden in der Türkei und schlussendlich auch von den Deutschen selbst, so dass er völlig isoliert ist.

Im Zuge dieser Überidentifizierung übernimmt Ömer auch den Deutschen zugeschriebene Charaktermerkmale, welche er in einer ins Krankhafte übersteigerten Form verkörpert. Es handelt sich vor allem um die Merkmale Pünktlichkeit und Rationalität. So hat Ömer eine ausgeprägte Zwangsneurose in Bezug auf Zeit, mit der er sich selbst und seine Familienmitglieder tyrannisiert. Er verlangt von seinen Familienmitgliedern, dass sie ihren ganzen Tagesablauf einem minutiös festgelegten Zeitraster unterwerfen und dass sie ihm immer wieder genaueste Angaben über die Uhrzeit machen, auch wenn dies völlig dys-

müsse, aus dem Gleichgewicht geraten. Das "ICH« "ist die organisierte Gruppe von Haltungen anderer, die man selbst einnimmt" (Mead 1973, 218). Ein gestörtes Verhältnis von Eigen- und Fremdwahrnehmung ist ein häufiges Phänomen in Texten türkischdeutscher Autoren, wie Dayıoğlu (2004) in einer erhellenden Analyse zur Integrität von Migranten-Figuren (besonders bei Aras Ören und Zafer Şenocak) darstellt. 
funktional sind. Ebenso notiert er in nur kurzen Abständen die genaue Uhrzeit von Erlebnissen und Handlungen in seinen Aufzeichnungen (beispielsweise Angaben über die Zeit des Händewaschens).

Abgesehen davon, dass Zeit und Pünktlichkeit mit dem sogenannten deutschen Nationalcharakter assoziiert werden, ist es noch aus einem anderen Grunde kein Zufall, dass Ömer ausgerechnet in Bezug auf Zeit eine Zwangsneurose entwickelt. Gemessene Zeit ist ein sehr präsentes Ordnungsraster, welches den sicherlich größten Einfluss auf die Strukturierung unseres modernen Lebens besitzt. Durch die Migration wird der Mensch aber aus den in seiner Kultur bestehenden, ihm vertrauten Rastern von Raum und Zeit herauskatapultiert und gezwungen, sich in neuen Raum-Zeit-Verhältnissen zurechtzufinden. Diese Anpassung gelingt Ömer anscheinend nicht, so dass in ihm das Gefühl entsteht, die Welt um sich herum und den zeitlichen Ablauf des Lebens nicht unter Kontrolle zu haben. Er hat ein sehr starkes Bedürfnis, eine äußerliche Ordnung herzustellen, da er die ihn umgebende Welt als chaotisch empfindet und auch in seinem Inneren diese Unordnung herrscht, der er durch die Zeithysterie Herr zu werden versucht.

Mit seinem Hauptanliegen - seine Notizen über die >Wahrheiten der Welt< zusammenzustellen und aufzuschreiben - vertritt Ömer außerdem die Seite des Wissens und der Rationalität, während demgegenüber seine Emotionen pathologisch verflacht sind, er steif, penibel, auch herablassend auftritt und unfähig zur Empathie ist, was sich vor allem in seinem Verhältnis zu seinen Familienmitgliedern manifestiert. In einer perversen Logik wirft aber Ömer diese Eigenschaften, die den Deutschen im allgemeinen Diskurs als nationale Charaktermerkmale zugeschrieben werden, den Deutschen vor, ohne zu merken, dass er sie selbst in viel stärkerer Weise verkörpert. Dies wird besonders gut in einer Szene entlarvt, in der Ömer in Pantoffeln in einen Zeitungskiosk geht, um die Zeitungen seiner >Journalistenkollegen $<$ zu kaufen. Als er den Kiosk betritt, macht eine der drei dort anwesenden Personen eine Bemerkung über seinen Aufzug, woraufhin Ömer gleich sein Ausländer- und Migrantendasein thematisiert (»wir Südländer«, EA, 146). Daraufhin wird er dann tatsächlich als Ausländer wahrgenommen (es findet eine projektive Identifizierung statt) und beleidigt (»Du hättest nicht herkommen sollen. Was hast du hier zu suchen?«, $E A, 146)$, und es entwickelt sich ein Disput, bei dem Ömer Mitleid erregend wirkt. Als er dann noch die Verkäuferin versehentlich mit »meine Mutter « anredet, tritt eine Kundin auf ihn $\mathrm{zu}$ und nimmt ihn ungestüm in ihre Arme: » Laß mich deine Mutter sein, mein Kleiner<, sagte sie.«(EA, 147) Die Deutsche zeigt hier also den Überschwang an Emotionen, den man sonst den Südländern zuschreibt. Ömer dagegen, der sich auch in dieser Szene wieder als warmblütigen Südländer bezeichnet und damit von den >steifen Deutschen< abgesetzt hatte, reagiert verletzt und beleidigt auf diesen Gefühlsausbruch. Trotzdem doziert er gegenüber den Anwesenden über die angebliche Wissensfixiertheit der Deutschen, welche auch ihr Verhältnis zu den Migranten bestimme, obwohl er gerade etwas anderes erlebt hat: 
Der Wissensschatz von euch Deutschen hat mich schon immer tief beeindruckt. Sie wissen soviel, daß Ihre Kenntnisse sich manchmal zwischen Sie und die Menschen schieben. [...] Auch Ihre Beziehungen zu den Ausländern sind davon mitgeprägt: Anstatt mit innen zusammenzuleben, halten Sie es für ausreichend, alle Arten von Kenntnissen über Sie zu erwerben. Das genügt natürlich nicht ... (EA, 151).

Ömers Beziehung zu Deutschland ist von einem unüberwindlichen Zwiespalt zwischen Verachtung und Unterwerfung geprägt sowie durch den zum Scheitern verurteilten Wunsch dazuzugehören, der ihn auch körperlich krankmacht: Magenschmerzen, Schlaflosigkeit, Atemnot, panische Angst und vieles mehr. Dass es sich hierbei letztlich um psychosomatische Beschwerden handelt, die Ausdruck der Angst vor einem Selbstverlust sind, der sehr deutlich im Zusammenhang mit der Migrationsproblematik steht, zeigt sich am Ende der hier beschriebenen Szene, als Ömer nach Hause geht und sagt: »Ich erstickte fast an der Angst, ich würde nie zu Hause ankommen, sondern bis in alle Ewigkeit verlorengehen.« $(E A, 151)$

\subsection{Die Doppelgängerproblematik auf der Vermittlungsebene als Ausdruck des Wahns}

Das Gefühl der Bedrohung durch angebliche böse Mächte, also der Verfolgungswahn, der Größenwahn und die Aufspaltung in verschiedene Persönlichkeiten, sind durchaus schizophrenietypisch; ${ }^{6}$ der Wahnsinn Ömers kann und soll indes nicht auf ein psychiatrisches Krankheitsbild reduziert werden, da die Doppelgängerthematik im weiteren Verlauf des Romans in weit komplexerer Weise ausgestaltet wird, so dass sich hier auf einer neuen Ebene der Zusammenhang von Identitätsproblematik und Migration zu erkennen gibt. Hierfür ist es notwendig, die Romanstruktur als Ganze und das Verhältnis der beiden Protagonisten - Ömer Kul im ersten Teil und Ibrahim Yaprak im zweiten Teil noch einmal genauer zu betrachten.

Anfänglich wurde gesagt, der Roman bestehe aus zwei Teilen, nämlich den beiden als jeweils eigene Romane markierten Zeitungstagen und den Notizen eines Spions. In Wirklichkeit jedoch - und dies übersieht man leicht - besteht der Text aus vier Teilen, denn jeweils am Ende der beiden Teil-Romane stehen einige Kapitel, in denen sich die beiden Figuren Ömer und Ibrahim im Krankenhaus treffen (EA, 206-216 u. 351-359) und welche das Verhältnis der beiden Teil-Romane in einem anderen Licht erscheinen lassen.

6 | Zwar gehören einige Merkmale der psychischen Störung Ömers in das Krankheitsbild der Schizophrenie (Verflachen der Affekte, Halluzinationen/Persönlichkeitsspaltung, Wahnideen wie Größenwahn und Verfolgungswahn), doch kann man seine Krankheit nicht eindeutig als schizophrene Störung einordnen (vgl. Andreasen/Black 1993; Weltgesundheitsorganisation 2010). Daher gebrauche ich die allgemeineren Begriffe 'Wahn und Persönlichkeitsstörung. 
Betrachtet man zunächst nur die beiden Teil-Romane, so ergeben sich zwei mögliche Interpretationen (s.o.): Erstens kann man Ibrahim als den halluzinierten Doppelgänger Ömers interpretieren. Für diese Version spricht einiges, da ja der Roman mit den Schilderungen des autodiegetischen Erzählers Ömer beginnt. Ömer leidet an einer wahnhaften Persönlichkeitsspaltung, den von ihm fantasierten Doppelgänger interpretiert er als einen ihn bedrohenden Spion. Seine eigenen Notizen schreibt er diesem Spion zu, sein Sohn holt die Notizen eines Spions aus Ömers Schublade, übergibt sie dem Arzt im Krankenhaus, und so gelangen sie wieder in die Hände Ömers. Insofern erscheint es auch logisch, dass der zweite Teil in Du-Form geschrieben ist, handelt es sich doch um eine Selbstansprache Ömers, die aus einer Schreibsituation der Halluzination und Identitätsspaltung heraus entstanden ist. Auch passt es ins Bild, dass Ömer sich im zweiten Teil selbst als Romanfigur bezeichnet, also als Fiktion, hat er sich doch längst in erfundene Personen aufgespalten und empfindet sich auch schon lange als nicht mehr verankert in seiner Realität.

Wenn Ömer in dem zweiten Teil als eine Romanfigur von Ibrahim Yaprak bezeichnet wird, so kann man den Roman aber auf eine zweite mögliche Art interpretieren, nämlich dahingehend, dass Notizen eines Spions der primäre Text ist, während Zeitungstage demgegenüber nur das Romanmanuskript des Schriftstellers Ibrahim wäre. Da Ömer Kul und Ibrahim sehr viele Parallelen aufweisen, kann man auch in dieser zweiten Lesart von einer DoppelgängerFantasie sprechen, was ich etwas genauer ausführen möchte, bevor ich auf die dritte mögliche Lesart zu sprechen komme.

Auch Ibrahim ist psychisch krank und leidet unter einer unsicheren Identität. Bei ihm manifestiert sich dies vor allem in Halluzinationen (EA, 251 u. 269), Auflösungserscheinungen (z.B. EA, 242 u. 351) und Ängsten, sich selbst zu verlieren. Diesen Tendenzen versucht er entgegenzuwirken, indem er sich selbst verletzt, um sich wieder als Ganzes zu spüren. Mit der ständigen Auflösung seines Körpers ist eine Vermischung von Traum und Wirklichkeit verknüpft: »Zu schlafen und dich in Alpträumen zu winden, die du vom wirklichen Leben schon nicht mehr zu unterscheiden weißt, ist dir zur unerträglichen Qual geworden.« (EA, 30of.) Auch in Bezug auf Ibrahim wird deutlich, dass diese Probleme in einem Zusammenhang mit der Migration stehen. Ibrahim fühlt sich stets als Ausländer, empfindet als Emigrant einen Rechtfertigungszwang, und er hat den Eindruck, nicht am eigentlichen Leben der Gesellschaft teilzunehmen. Dies ruft - wie auch bei Ömer - einen unerträglichen Zwiespalt in ihm hervor, zumal er auch keinen Zugang mehr zu seiner Vergangenheit und seinen alten Gefühlen findet bzw. diese nicht in seine Gegenwart integrieren kann. Dies wird in einer Szene deutlich, als Ibrahim plötzlich von unerklärlichen Hungerattacken befallen wird, auf die er mit exzessivem Kochen reagiert, wobei er am Ende das Essen jedoch gar nicht zu sich nehmen will. Entscheidend ist hier der Kochvorgang selbst, denn die dabei entstehenden Gerüche lassen »[a]lle möglichen verdrängten Erinnerungen« $(E A, 316)$ in ihm wach werden. Gerüche, welche, wie die Traumaforschung herausfinden konnte, den direktesten und unkontrollierbarsten Zugang zu Erinnerungen liefern, also besonders 
starke Trigger für die Erinnerung sind (vgl. z.B. Fischer/Riedesser 2009), sind für Ibrahim ein Versuch, um eine Verbindung zwischen Vergangenheit und Gegenwart herzustellen, was ihm jedoch nur selten gelingt.

Sowohl die psychosomatischen Krankheitssymptome als auch viele andere psychische Probleme Ibrahims ähneln denen Ömers (z.B. Schmerzen, Atemnot, Ängste, das Vermischen von Traum und Realität, das Zeitproblem). Während dies aber bei Ömer ausschließlich als Folge der nicht bewältigten Migration und eines Minderwertigkeitskomplexes als (>Gast<-)Arbeiter dargestellt wird, könnte man Ibrahims psychische Probleme außerdem als Folge eines Verfolgungstraumas deuten. Der Intellektuelle Ibrahim ist als politischer Flüchtling nach Berlin gekommen, und es gibt einige Hinweise darauf, dass er in der Türkei einer dramatischen politischen Verfolgung ausgesetzt war (z.B. EA, 244, 265 u. 319). Seine politisch revolutionären Ideen leben bis in die Gegenwart fort, und er leidet darunter, dass er sie in Deutschland nie realisieren konnte. Die Ängste, der Verfolgungswahn, die psychosomatischen Krankheiten, die Persönlichkeitsdissoziation, das Verwischen von Fiktion und Realität passen zu der Symptomatik einer posttraumatischen Störung, die nicht bewältigt wurde. Außerdem gehören die von Ömer nicht geteilten Symptome Sucht (Ibrahim ist Alkoholiker) und Selbstverletzung in dieses Krankheitsbild.7 Aufgrund der beschriebenen Umstände kann der Migrant Ibrahim in seiner neuen Lebensrealität nicht Fuß fassen, sondern lebt seit seiner Auswanderung mit der Lebenslüge seines angeblichen Romans. In dem Moment, als er das Projekt tatsächlich realisieren will, bricht der jahrelang mühsam aufrechterhaltene psychische Schutzwall zusammen und er wird wahnsinnig. Je intensiver sich Ibrahim mit dem Roman beschäftigt, umso mehr gerät er psychisch aus dem Gleichgewicht und muss feststellen, dass seine Balance zwischen Fantasie und Wirklichkeit verloren geht. So wie für Ömer die Zeitung immer mehr zu einer eigenen Realität wird, verselbständigen sich auch Ibrahims Romanprojekt und seine Romanfigur Ömer:

7 | Während für die Schizophrenie eine Persönlichkeitsspaltung typisch ist, bei der der Erkrankte zwei gleichzeitige Persönlichkeiten ausbildet, findet bei der posttraumatischen Störung eine Dissoziation in verschiedene Persönlichkeiten statt, welche die Betroffenen abwechselnd und nacheinander, aber nicht gleichzeitig einnehmen. Für die Einordnung in das Krankheitsbild einer posttraumatischen Störung sprechen außerdem folgende Symptome Ibrahims, welche nicht bei Ömer auftreten: das Suchtverhalten (er ist Alkoholiker) und die Selbstverletzung (vgl. Fischer/Riedesser 2009; Weltgesundheitsorganisation 2010). Ibrahim leidet dagegen nicht unter Verfolgungswahn, welcher wiederum schizophrenietypisch ist. Eine genaue psychiatrische Diagnostik der Krankheiten von Ömer und Ibrahim wird hier nicht angestrebt, da sie dem Text als literarischer Fiktion nicht gerecht wird. Es ist jedoch erstaunlich, welch feine Unterscheidungen der Text in Bezug auf die Erkrankung, ihre Symptome und ihre Ursachen macht, welche eine relativ genaue psychiatrische Einordnung zulassen. 
Nun hast du so viele Jahre nach einer Geschichte gesucht, die innerlich und äußerlich mit dir im Einklang steht, hast sie so lange im Kopf reifen lassen, und da kommt jetzt ein gewisser Ömer daher, dessen genaue Identität noch gar nicht feststeht und versucht auch noch, dich von deinem Weg abzubringen, bemüht sich, dich an dir selbst, an deinem Vertrauen in dich selbst zweifeln zu lassen ... [...] Beim Frühstück erklärtest du deiner Frau, Ömer habe die Geschichte akzeptiert. Dazu sei jedoch dein Drängen notwendig gewesen, denn er habe, soweit du es beobachten konntest, die Geschichte nicht mal in ihren groben Zügen begriffen. (EA, 335f.)

Beide Projekte und beide Doppelgängerfiguren - Zeitung und Roman, Ibrahim und Ömer - verselbständigen sich zu einer imaginierten Realität und werden zu einer fixen Idee, die den latent vorhandenen Wahnsinn der beiden Figuren manifest werden lässt. Ömer und Ibrahim können als ein und dieselbe Figur betrachtet werden, über die ihre psychische Störung Macht ausübt: »Seit Tagen schreibst du da etwas, anscheinend nicht aus eigener Kraft, sondern als ob ein anderer, eine mysteriöse Kraft, die die Kontrolle über dich erlangt hat, es dir diktiert ...«, sagt Ömer $(E A, 165)$. Die Identität der beiden Figuren wird untermauert durch zahlreiche im Text verstreute Hinweise darauf, dass sich der erste und der zweite Romanteil gleichzeitig ereignen. Mehrere Szenen und Ereignisse des ersten Teils werden im zweiten Teil wiederholt, zunächst aus dem Blickwinkel Ömers, später aus demjenigen Ibrahims, dabei aber mit leichter Abwandlung. Es stellt sich die Frage, welche Figur, also welcher Teil des Romans der primäre ist.

Die Ausführungen zu der möglichen zweiten Lesart und der Identitätsproblematik der zweiten Figur zeigen bereits, dass in dem Roman ein Verwirrspiel mit den Fiktionsebenen getrieben wird. Dieses wird mit der dritten möglichen Lesart des Textes auf die Spitze getrieben, welche sich in den Passagen im Krankenhaus, jeweils am Ende der beiden Romanteile, entfaltet. Diese Passagen sind in einem anderen Erzählmodus gestaltet als die beiden Romanteile. Die Begegnungen zwischen Ömer und Ibrahim werden im dramatischen Modus mitgeteilt, es handelt sich um eine Wiedergabe von Dialogen zwischen den beiden Figuren bzw. zwischen ihnen und dem Arzt ohne Verba dicendi. Ömer und Ibrahim treten nun nicht als Fiktionen des jeweils anderen auf, sondern vielmehr als gleichberechtigte Figuren in einem Roman, für den man einen übergeordneten Erzähler annehmen muss. Die beiden Figuren treffen sich, sprechen miteinander, wundern sich über die Existenz des jeweils anderen und befreunden sich. Ibrahim versucht am Ende mit allen Mitteln, Ömer bei sich zu behalten, da er sich ohne ihn einsam fühlt.

Formal wird für diese Passagen ein übergeordneter Erzähler auf den letzten beiden Seiten des Romans suggeriert, da sich nämlich Ibrahim als der Autor des Romans Der enthaarte Affe bezeichnet. Er gibt Ömer ein Manuskript zu lesen, welches aus den Zeitungstagen und den Aufzeichnungen eines Spions besteht und welches nun von Ömer (und mit ihm vom textexternen Rezipienten) in einer neuen, nicht-linearen Reihenfolge gelesen werden soll, die im letzten Kapitel genannt wird und auch schon dem Roman zu Beginn als Wegweiser vorangestellt ist. Trotz dieses Romanschlusses bleibt die entscheidende Frage, 
welche der beiden Figuren die textintern reale und welche die imaginäre ist, unaufgelöst und unaufösbar, da die Paradoxien auf der Inhaltsebene bestehen bleiben. Die Tatsache, dass auch Ömer als primärer Erzähler lesbar ist, bleibt weiter bestehen. Ibrahim besetzt zwar die Position des Textarrangeurs, doch wird er auch ganz am Ende wieder in der zweiten Person angesprochen, so dass es formal noch eine Erzählinstanz geben muss, die über ihm steht. Es spricht auch weiterhin vieles dafür, dass Ömer in Form einer Selbstansprache derjenige ist, welcher Ibrahim mit Du anredet, zumal es heißt, dass beide Romanteile in Ömers Handschrift verfasst sind. Dann aber könnte Ibrahim ja nicht der übergeordnete Erzähler sein. Das Hierarchieverhältnis zwischen den beiden Figuren sowie die Frage nach dem eigentlichen übergeordneten Erzähler lässt sich nicht abschließend klären, da die beiden Figuren zwei Seiten einer gespaltenen Persönlichkeit sind und sich kein verlässlicher, übergeordneter Erzähler zu erkennen gibt, der den Rezipienten an die Hand nimmt. Es existiert vielmehr ein unentwirrbares Spiel mit den Fiktionsebenen, bei dem die Grenzen von Realität und Fiktion überschritten werden.

Diese Unentscheidbarkeit, die Unauflösbarkeit von Wahn und Realität, die Unentwirrbarkeit der Fiktionsebenen, die Verselbständigung von Romanfiguren, Fiktionen und Wahnideen - all dies interpretiere ich als Ausdruck einer interkulturellen Auseinandersetzung Dals in seinem Roman, in dem sich die Semantik auf der Ebene der Form wiederholt. So wie Ömer und Ibrahim einsam, ausgeschlossen, verwirrt und gespalten bzw. dissoziiert sind, keine Linie im Leben, keine haltbare Selbstdefinition haben und die Raum- und Zeit-Koordinaten gestört sind, so gestaltet sich auch die Textstruktur als räumlich und zeitlich gestörter Komplex, der keine eindeutige Auflösung ermöglicht. Sind für die beiden Hauptfiguren die Abgrenzungen zwischen Welt und Ich, Traum und Wirklichkeit nicht eindeutig, so ist diese Grenzauflösung auch strukturell im Text markiert, kann man doch nicht entscheiden, wer wessen Erfindung ist, wer real und wer fiktiv ist. Die migrationsbedingt gespaltene Persönlichkeit, die in ihrer Identität verunsichert ist und ihre Teile nicht mehr zusammenbekommt, spiegelt sich auch in der Struktur des Romans wider. Dem im modernen Diskurs immer wieder unter positiven Vorzeichen erwähnten Konzept der kulturellen Identität, nach dem das moderne Individuum frei gewählte Identitätsteile gewinnbringend zu einer hybriden, aber stabilen Identität $\mathrm{zu}$ integrieren weiß, wird hier als unerreicht oder gar unerreichbar eine Absage erteilt. ${ }^{8}$

8 | Das Konzept der kulturellen Identität wurde vor allem von Stuart Hall (1996) in die Diskussion gebracht und wird nicht nur in der interkulturellen Literaturwissenschaft immer wieder aufgegriffen. Nach Hall könne das moderne Individuum keine statische Identität mehr ausbilden, wie sie von Erikson (1973) noch beschrieben wird, sondern müsse sich Passendes aus verschiedenen Bereichen zusammensuchen. Kritiker dieser Annahme führen ins Feld, dass der Mensch das Bedürfnis nach Stabilität und einer geordneten Welt um sich herum habe und ein solch hybrides Identitätskonzept wohl nur theoretisch funktioniere. Auch Dayıoğlu $(2004,109)$ beobachtet bei den Romanfiguren deutsch-türkischer Autoren in erster Linie einen Kampf um "Integrität", um 


\section{SCHLUSSBEMERKUNGEN}

Eingangs wurde geschildert, dass die ersten Texte von türkischen Emigranten (>Gastarbeitern $<$ ) in Deutschland die Themen der Ankunft in der Fremde und der Gastarbeit in betont schlichten, reportageartigen Texten verarbeiteten. Dals Text bricht mit dieser Entwicklung, indem für ihn ein neues Thema wichtig wird - nämlich die migrationsbedingte Identitätsproblematik und die interkulturelle Auseinandersetzung, welche für den schon lange in Deutschland lebenden >Gastarbeiter < relevant werden - und indem er ein starkes Gewicht auf die Vermittlungsebene legt. Die literarische Verfremdung stellt hier insofern eine besondere Verflechtung von Form und Inhalt dar, als durch die hervorgekehrte Erzählweise zum einen ein Moralisieren und Belehren (wie wir es zum Teil in den frühen Texten vorfinden) ${ }^{9}$ vermieden werden kann, zum anderen die notwendige Distanz geschaffen wird, um sensible Themen bearbeiten zu können, ohne Vorurteile und Hass zu schüren. ${ }^{10}$ Dal stellt somit ein Bindeglied zwischen den frühen, damals als »Gastarbeiterliteratur « bezeichneten Texten und denen der heute bekanntesten Autorinnen und Autoren wie Emine Sevgi Özdamar oder Feridun Zaimoğlu dar. Während Letztere von einer übernationalen, kosmopolitischen Perspektive aus schreiben und teilweise bereits die Anliegen der Kindergeneration fokussieren," greift Dal in seinem komplexen Text noch einmal ein Thema aus der ersten Einwanderergeneration auf, wenn er zwei Migrationsschicksale und zwei exemplarische Sozialtypen präsentiert, nämlich den >Gastarbeiter Ömer und den politischen Oppositionellen Ibrahim.

eine innere Übereinstimmung mit sich selbst, statt einer positiven Ausdifferenzierung. Kaputanoğlus (2002) positive Bewertung der hybriden Identität der Romanfiguren Ömer und Ibrahim kann ich dagegen nicht nachvollziehen.

9 | Die einfache Gestaltung in den frühen Texten der türkischen Autoren in Deutschland hat oft eine Schwarz-Weiß-Zeichnung der Charaktere und ein Oppositionen schürendes Moralisieren zur Folge (vgl. z.B. Savaşçı 1983 u. 1987).

10 | Auch Hofmann $(2006,59 f$.) teilt die Beobachtung, dass die interkulturelle Auseinandersetzung häufig eine starke Hervorkehrung literarischer Stilmittel mit sich bringt. Er nennt als Beispiele Satire, Parodie, Groteske, Fantastik und Absurde.

11 | Vgl. bes. Zaimoğlu 1995; das Buch präsentiert literarisch stilisierte "Protokolle" von türkisch-deutschen Jugendlichen, in denen diese ihre Identitätsfindung in Abgrenzung von der deutschen Gesellschaft und ihren ('Gastarbeiter-)Eltern beschreiben. 


\section{LITERATUR}

Ackermann, Irmgard/Weinrich, Harald (Hg., 1986): Eine nicht nur deutsche Literatur. Zur Standortbestimmung der "Ausländerliteratur». München

Andreasen, Nancy C./Black, Donald W. (1993): Lehrbuch Psychiatrie. Übers. v. Diana Horn, Ralf Horn u. Ellinor Schreiner. Weinheim/Basel

Blumentrath, Hendrik u.a. (2007): Transkulturalität: Türkisch-deutsche Konstellationen in Literatur und Film. Münster

Chiellino, Carmine (Hg., 2000): Interkulturelle Literatur in Deutschland. Ein Handbuch. Stuttgart

Dal, Güney (1988): Der enthaarte Affe. Roman. Übers. v. Carl Koß. München

Ders. (1999): Janitscharenmusik. Roman. Übers. v. Carl Koß. München

Dayıoğlu, Yasemin (2004): Von der Gastarbeit zur Identitätsarbeit - der Kampf um Integrität in der Migrationsliteratur. In: Manfred Durzak/Nilüfer Kuruyazycy (Hg.): Die randere، Deutsche Literatur. Istanbuler Vorträge. Würzburg, S. 104-110

Durzak, Manfred/Kuruyazıcı, Nilüfer (Hg., 2004): Die sandere، Deutsche Literatur. Istanbuler Vorträge. Würzburg

Erikson, Erik H. (1973): Identität und Lebenszyklus. Drei Aufsätze. Übers. v. Käte Hügel. Frankfurt a.M.

Fischer, Gottfried/Riedesser, Peter (2009): Lehrbuch der Psychotraumatologie. München/Basel

Hall, Stuart (1996): The Question of Cultural Identity. In: David Held/Tony McGrew/ Stuart Hall (Hg.): Modernity and its Futures. Understanding Modern Societies. Cambridge, S. 274-316

Hamm, Horst (1988): Fremdgegangen - Freigeschrieben. Einführung in die deutschsprachige Gastarbeiterliteratur. Würzburg

Hofmann, Michael (2006): Interkulturelle Literaturwissenschaft. Eine Einführung. Paderborn

Kaputanoğlu, Anıl (2002): Zur Neuformierung des Gedächtnisses in Repräsentationen des Anderen am Beispiel von Güney Dals Roman "Janitscharenmusik" (1999) in der Übersetzung von Carl Koß. In: Dies./Nicole Meyer (Hg.): "Nur das Auge weckt mich wieder ...". Erinnerung - Texte - Gedächtnis. Münster, S. 69-88

Kassouf, Susan (1994): "Wir haben innen Romanautoren gesandt«: Aras Ören's "Eine verspätete Abrechnung" und Güney Dal's "Der enthaarte Affe". In: Carol Aisha Blackshire-Belay (Hg.): The Germanic Mosaic. Cultural and Linguistic Diversity in Society. Greenwood, S. 57-62

Keupp, Heiner u.a. (Hg., 2008): Identitätskonstruktionen. Das Patchwork der Identitäten in der Spätmoderne. Reinbek b. Hamburg

Kora, Kaan/Özek, Metin/Baral-Kulaksızoğlu, Işın (1998): Ein Beitrag zum Thema der wahnhaften Depressionen (mit Fallbeispielen aus der Türkei). In: Eckhardt Koch u.a. (Hg.): Chancen und Risiken von Migration. Deutsch-türkische Perspektiven. Freiburg i.Br., S. 181-185

Kreuzer, Helmut (1984): Gastarbeiter-Literatur, Ausländer-Literatur, Migranten-Literatur? Zur Einführung. In: Ders./Peter Seibert (Hg.): Zeitschrift für Literaturwissenschaft und Linguistik, 14, Sonderh. 56: "Gastarbeiterliteratur", S. 7-11 
Lorenz, Günter W./Pazarkaya, Yüksel (Hg., 1985): ... aber die Fremde ist in mir. Migrationserfahrung und Deutschlandbild in der türkischen Literatur der Gegenwart. Stuttgart

Mead, George Herbert (1973): Geist, Identität und Gesellschaft aus der Sicht des Sozialbehaviorismus. Hg. u. mit e. Einl. v. Charles W. Morris. Übers. v. Ulf Pacher. Frankfurt a.M.

Ören, Aras (1973): Was will Niyazi in der Naunynstraße. Ein Poem. Übers. v. H. Achmed Schmiede u. Johannes Schenk. Berlin

Pazarkaya, Yüksel $(1982,1992)$ : Rosen im Frost. Einblicke in die türkische Kultur. Zürich

Savaşçı, Fethi (1983): Bei laufenden Maschinen. In: Ders.: Bei laufenden Maschinen/Makinalar Çalişirken. Erzählungen/Öyküler. Deutsch/Türkçe. Übers. v. Helga Dağyeli-Bohne u.a. Frankfurt a.M., S. 16-20

Ders. (1987): Mayer war sehr verärgert. In: Ders.: München im Frühlingsregen. Erzählungen und Gedichte. Übers. v. Zafer Şenocak. Frankfurt a.M., S. 8-12

Weigel, Sigrid (1992): Literatur der Fremde - Literatur in der Fremde. In: Klaus Briegleb/ Sigrid Weigel (Hg.): Gegenwartsliteratur seit 1968. München/Weimar, S. 182-229

Weltgesundheitsorganisation (2010): Internationale Klassifikation psychischer Störungen. ICD-10 Kapitel V (F): Klinisch-diagnostische Leitlinien. Hg. u. übers. v. H. Dilling, W. Mombour u. M.H. Schmidt. Bern

Yeşilada, Karin E. (2000): Topographien im "tropischen Deutschland " - Türkisch-deutsche Literatur nach der Wiedervereinigung. In: Ursula E. Beitter (Hg.): Literatur und Identität. Deutsch-deutsche Befindlichkeiten und die multikulturelle Gesellschaft. New York, S. 303-339

Dies. (2008): Türkischdeutsche Literatur. In: Tayfun Demir (Hg.): Türkischdeutsche Literatur. Chronik literarischer Wanderungen. Duisburg, S. 11-15

Zaimoğlu, Feridun (1995): Kanak Sprak. 24 Mißtöne vom Rande der Gesellschaft. Hamburg 